\title{
Microwave-assisted synthesis of anion-exchange resins for sorption of noble metals: how to boost sorption capacity using a proper reaction environment
}

\author{
Dorota Jermakowicz-Bartkowiak ${ }^{1}$. \\ Piotr Cyganowski ${ }^{1} \cdot$ Jarosław Kawałko $^{1}$
}

Received: 28 January 2016/Revised: 7 April 2016/Accepted: 30 May 2016/

Published online: 3 June 2016

(C) The Author(s) 2016. This article is published with open access at Springerlink.com

\begin{abstract}
In the present studies a series of anion-exchange resins was synthesized in a microwave field. The 1,6-diaminohexane functionalized resins were obtained in presence of selected organic solvents, $N, N$-dimethyl formamide, dimethyl sulfoxide and 1-methyl-2-pyrrolidone. The resins were employed in batch and dynamic processes of $\mathrm{Au}(\mathrm{III}), \mathrm{Pt}(\mathrm{IV})$ and $\mathrm{Pd}(\mathrm{II})$ sorption from tricomponent systems in $0.1 \mathrm{M}$ $\mathrm{HCl}$. The experiment was designed in a way that allowed to determine the use of a specific solvent in the microwave field and how it impacts on properties of the anion exchangers. An influence of a reaction environment was discussed taking into account i.e., dielectric characteristics of the specific solvent, efficiency of the syntheses processes as well as the maximum sorption capacity of the resins. Ultimately the application of a specific reaction environment was set together with sorption of noble metals ability and evaluated using infrared spectroscopy. The proceeded analyzes allowed to determine which organic solvent from the selected ones is the most suitable for microwave-assisted synthesis of the anion-exchange resins.
\end{abstract}

Keywords Microwaves · Anion-exchange $\cdot$ Gold $\cdot$ Platinum $\cdot$ Palladium

\section{Introduction}

Heating is the primary stimulant of chemical reactions that proceed slowly or do not occur at all under ambient conditions. Within last few decades a number of techniques for enhancing the rate of the processes have been developed and used. Among them reactions induced or improved by applying photo-, ultrasonic-,

Piotr Cyganowski

piotr.cyganowski@pwr.edu.pl

1 Division of Polymer and Carbonaceous Materials, Faculty of Chemistry, Wroclaw University of Technology, Wybrzeże Wypisańskiego 27, 50-370 Wrocław, Poland 
plasma- and microwave-chemistry can be distinguished. Microwave heating is one of the most interesting. It uses the ability of specific solids and liquids to transform electromagnetic energy into heat. As a result, the energy conversion acts on a molecular scale and creates attractive applications in chemistry [1].

In many cases microwave-assisted processing of materials has significant advantages over traditional techniques [1-3]. That includes more precise and volumetric heating, lower energy consumption and much shorter time required for completing the reactions $[1,4,5]$.

Microwaves are part of the electromagnetic spectrum with a length of $1 \mathrm{~mm}$ to $1 \mathrm{~m}$, which corresponds to a frequency of $0.3-300 \mathrm{GHz}$. Microwave radiation is non-ionizing and transmits energy through interaction with polar substances.

Microwaves cause rotation of polar molecules in the alternating electric field; therefore, a substance must be characterized by a constant dipole moment. Those dipoles orient themselves rotating in the area of radiation impact, matching with its direction. Then, created in that way energy is being lost in molecular frictions and collisions, resulting with the heat $[1,6]$.

Microwave energy is dissipated by three different mechanisms: magnetic losses in the ferromagnetic materials, ohmic losses in the conductive materials and the electrical losses attributed to the heterogeneity of the electromagnetic ions or molecules with a dipole moment. From the practical point of view, the materials, that dipole moment is greater than zero are particularly important. They have ability to absorb electromagnetic radiation, entirely or partially, and transform it into heat. If a specific reagent cannot interact with the microwave field (its dipole moment is equal to zero) it can capture the energy from an applied reaction environment. For that reason selection of a suitable solvent for the microwave-assisted synthesis is essential [6].

Organic solvents may be characterized by two factors, relevant from the point of view of microwave-enhanced chemistry: dielectric constant $\left(\varepsilon^{\prime}\right)$ that is a measure of the ability of a substance to store electric charges and dielectric loss $\left(\varepsilon^{\prime \prime}\right)$, absolute electric permeability, the amount of electromagnetic energy, that a matter converts into the heat. These two parameters are related with a tangent of a loss angle (1) [1]:

$$
\tan \delta=\frac{\varepsilon^{\prime \prime}}{\varepsilon^{\prime}}
$$

The parameter defines the ability of a substance, treated with the microwave field, to transform electromagnetic energy into heat at a given temperature and power of the radiation. In fact, the greater value of $\tan \delta$ is, the more efficient microwave-heating can be observed $[1,6]$.

In our previous work [7] we have reported a rapid procedure of microwaveassisted synthesis of the new amino anion-exchange resins. Proceeded tests on sorption of gold, platinum and palladium revealed that the resins obtained in the microwave field were equal to those, synthesized using the traditional techniques. The materials become more attractive, after considering the fact, that microwaveassisted process has taken 1500 times less time than the most efficient, conventional one. 
Because applied for the synthesis copolymer of vinylbenzyl chloride (VBC) and divinylbenzene (DVB), VBC/DVB, does not interact with microwaves it was necessary to apply a suitable reaction environment. As a result, synthesis was proceeded in $\mathrm{N}, \mathrm{N}$-dimethylformamide (DMF) using amines as functionalizing agents. Executed analyzes revealed that the microwaves facilitate creation, instead of amino functional groups, the $-\mathrm{C}=\mathrm{N}-$ bonds, derived from $\mathrm{DMF}$, resulting with smaller sorption capacity of the received anion exchangers [7, 8].

The worldwide literature does not provide any information about the influence of reaction environment on the properties of anion exchangers obtained in the microwave field. For that reason, it is necessary to find out, how the selection of an organic solvent influences the sorption ability of the final products, and what determines its usability.

The objective of the present work is to access a suitability of selected organic solvents for microwave-assisted synthesis of anion exchange resins. The studies covered changes in the anion exchangers chemical structures as well as modification efficiency. Then, these polymers were evaluated in practical terms applying them in the processes of sorption of $\mathrm{AuCl}_{4}{ }^{-}, \mathrm{PtCl}_{6}{ }^{2-}$ and $\mathrm{PdCl}_{4}{ }^{2-}$ chlorocomplexes from solutions in $0.1 \mathrm{M} \mathrm{HCl}$. Evaluation of the uptake of gold platinum and palladium, as well as considerations on modification procedure itself, allowed to determine the correlation between applied reaction environment and characteristics of the obtained in the microwave field anion exchange resins. Furthermore, it was possible to determine what factor states the usability of a selected solvent for the microwaveassisted process.

\section{Methods and materials}

\section{Selection of organic solvents}

To access an influence and the usability of the applied reaction environment it was necessary to select organic solvents varied by different dielectric constant $\varepsilon^{\prime}$ and dielectric loss $\varepsilon^{\prime \prime}$ that are furthermore, suitable for microwave-assisted processes (are polar substances).

For that reason $\mathrm{N}, \mathrm{N}$-dimethyl formamide (DMF), dimethyl sulfoxide (DMSO) and 1-methyl-2-pyrrolidone (NMP) were applied. Table 1 displays the characteristics of these solvents relevant for the utilized synthesis procedure [6].

\section{Reagents}

The organic solvents, N,N-dimethyl formamide (DMF), dimethyl sulfoxide (DMSO) and 1-methyl-2-pyrrolidone (NMP) used as the reaction environment as well as reagents used for characterisation of the obtained ion-exchange resins were bought in Avantor Performance Materials Inc. (former POCh) and used as received.

The 1,6-diaminohexane (DAH) applied for modification of the initial polymeric matrix, VBC/DVB, was purchased in Sigma-Aldrich Chemical Company and used as received. 
Table 1 Characteristics of the applied organic solvents

\begin{tabular}{llllll}
\hline Solvent & $\mathrm{T}_{\mathrm{B}}^{\mathrm{a}}$ & $\varepsilon^{\prime \mathrm{b}}$ & \multicolumn{1}{c}{$\varepsilon^{\prime \prime \mathrm{c}}$} & $\tan \delta^{\mathrm{d}}$ & $\mathrm{A}^{\mathrm{e}}$ \\
\hline DMF & 154 & 37.7 & 6.07 & 0.161 & Middling \\
DMSO & 189 & 45.0 & 37.13 & 0.825 & Good \\
NMP & 204 & 32.2 & 8.86 & 0.275 & Middling \\
\hline
\end{tabular}

\footnotetext{
${ }^{\text {a }}$ Boiling temperature $\left({ }^{\circ} \mathrm{C}\right)$

b Dielectric constant

c Dielectric loss

d Tangent of a loss angle

e Ability to absorb the microwaves
}

Solutions applied for studies on sorption were prepared by dissolving of $\mathrm{HAuCl}_{4} \cdot 4 \mathrm{H}_{2} \mathrm{O}$, and diluting solutions of $\mathrm{H}_{2} \mathrm{PtCl}_{6}$ and $\mathrm{H}_{2} \mathrm{PdCl}_{4}$ in $0.1 \mathrm{M} \mathrm{HCl}$, respectively. These acids were acquired from Avantor Performance Materials Inc. (gold) and INNOVATOR Ltd. (platinum and palladium).

\section{Synthesis procedure}

The initial, expanded-gel structure VBC/DVB (2\%) copolymer, used as a matrix for the ion-exchange resins, was obtained in the presence of toluene using the procedure previously described in the reference cited in [9].

Then, about $3 \mathrm{~g}$ of the received VBC/DVB copolymer was swollen overnight in reaction glass tube, in $30 \mathrm{~cm}^{3}$ of DMF, DMSO or NMP, respectively. Next, DAH was added in amount that allowed to keep molar ratio 3:1 to prevent spontaneous post-crosslinking during modification process.

Glass tubes with prepared mixtures of an amine and the swollen copolymer, were placed in the ERTEC Microwave Reactor 02-02 equipped with a reflux condenser and magnetic stirrer.

Power of microwaves must have been cleverly chosen. It could not be too great, because there was a risk that the reaction mixture might reach the set temperature limit too fast resulting with cutting-off the microwave field. If that happens, the further modification should be considered as conventional. In that circumstances an assessment of the influence of the microwaves could be difficult.

Of course, the power of the microwave field might have been set in a way that could ensure reaching the boiling point of the specific solvent. Without doubt the reaction proceeded in that conditions would be extremely efficient. However, there was a risk that properties of the synthesized anion exchange resins could be attributed not only to the microwaves but to the temperature of the modification as well.

Considering the above mentioned issues and observing the behavior of the solvents in the microwave field, it was decided to set the power of the microwaves at $40 \mathrm{~W}$. Application of the same, low power for all of the processes ensured, on the one hand, excluding the risk of a premature shutdown, on the other, determining the influence of microwaves themselves on the modification process. Furthermore, the 
selected power allowed for minimizing the effect of the temperature of the syntheses, and if any differences occur, they will be attributed strictly to the properties of the solvent indicating its usability.

Summarizing, the modification was proceeded within $15 \mathrm{~min}$ in the microwave field set at $40 \mathrm{~W}$. The temperature of the processes was considered only as secondrate factor, nevertheless, its value was constantly monitored. The recorded during the process parameters are displayed in the Fig. 1.

\section{Getting the anion exchange resins ready to work}

The microwave-assisted process resulted with synthesis of three anion exchange resins. The polymers $1 \mathrm{D}, 1 \mathrm{~S}$ and $1 \mathrm{~N}$, with functionalities derived from DAH were obtained in the presence of DMF (the resin 1D), DMSO (the resin 1S) or NMP (the

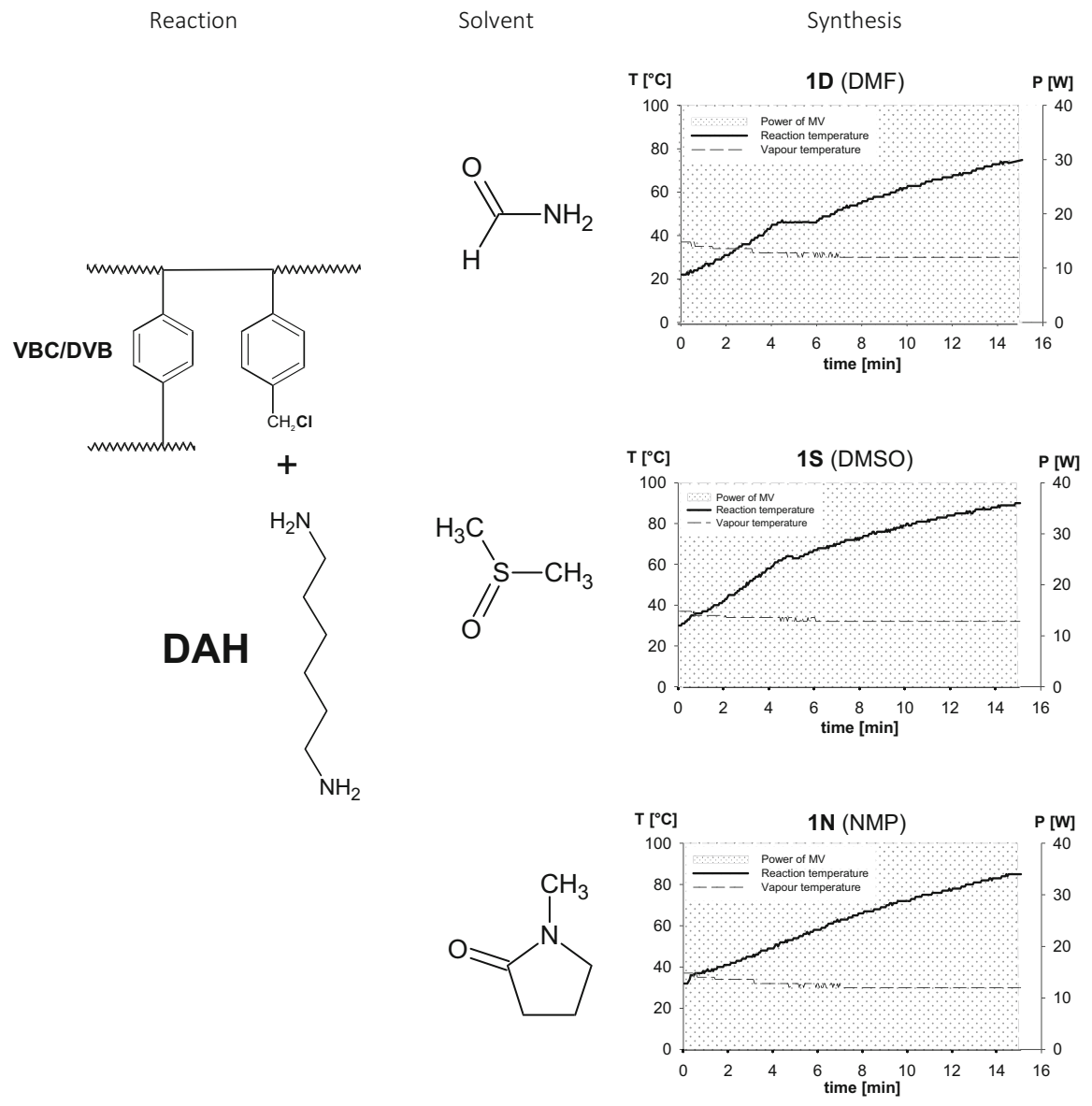

Fig. 1 The actual recorded parameters of the syntheses set together with chemical structures of the reagents 
resin $1 \mathrm{~N}$ ), respectively. After syntheses the resultant products were placed on a frittered-glass funnel and washed successively using cold and hot distilled water to remove the remaining solvents, unreacted amine.

Then, the anion exchange resins were packed into ion exchange columns and washed through with distilled water, $1 \mathrm{M} \mathrm{HCl}$, distilled water again and $1 \mathrm{M} \mathrm{NaOH}$. Finally, the resins were washed using $1 \mathrm{M} \mathrm{HCl}$ and $0.001 \mathrm{M} \mathrm{HCl}$ and as such used for further procedures.

\section{Characterization of the anion exchange resins}

The $0.001 \mathrm{M} \mathrm{HCl}$ regain was measured using centrifugation technique [10]. The nitrogen and chlorine content were determined in accordance to the Kiejdahl's $[11,12]$ and Schöniger's [13] methods, respectively. Hecker's procedure was applied for determination of the anion exchange capacity, $Z_{H}$. [14].

The FTIR spectra in KBr pallets were recorded using Perkin-Elmer System 2000 spectrophotometer. The results of the analyzes were collected in the Table 2 and discussed in "Results and discussion".

\section{Sorption studies}

Ability of the synthesized anion exchangers for sorption of $\mathrm{AuCl}_{4}{ }^{-}, \mathrm{PtCl}_{6}{ }^{2-}$ and $\mathrm{PdCl}_{4}{ }^{2-}$ was evaluated using two multicomponent solutions in $0.1 \mathrm{M} \mathrm{HCl}$ where metals were present in concentration of $0.24 \mathrm{mM}$ (solution A) and $4.80 \mathrm{mM}$ (solution B) of each metal.

The systems were composed using the following procedure: sorption tests from the solution $\mathrm{A}$ were designed in a way that keeps molar ratio of amino functionalities (estimated by $Z_{\mathrm{H}}$, Table 2 ) to the metals' chlorocomplexes at 10:1. The ratio during sorption tests from the solution $B$ was kept at 1:3.

First case (solution A) will let to determine if the synthesized resins are able to remove the noble metals completely. Second one (solution B), where all of the functionalities should be saturated, will indicate the maximum sorption capacity as well as a selectivity towards a specific metal.

Table 2 Conditions of synthesis and characteristics of the resultant products

\begin{tabular}{llllllll}
\hline Resin & Solvent & $\mathrm{W}^{\mathrm{a}}$ & $\mathrm{Cl}^{\mathrm{b}}$ & $\mathrm{Cl}^{\mathrm{c}}$ & $\mathrm{Cl}^{\mathrm{d}}$ & $\mathrm{N}^{\mathrm{e}}$ & $\mathrm{Z}_{\mathrm{H}}^{\mathrm{f}}$ \\
\hline $1 \mathrm{D}$ & DMF & 0.86 & 0.32 & 4.38 & 4.06 & 4.23 & 3.05 \\
$1 \mathrm{~S}$ & DMSO & 1.03 & 0.32 & 4.55 & 4.23 & 4.69 & 3.13 \\
$1 \mathrm{~N}$ & NMP & 1.72 & 0.41 & 4.73 & 4.32 & 5.25 & 3.56 \\
\hline
\end{tabular}

${ }^{\mathrm{a}} 0.001 \mathrm{M} \mathrm{HCl}$ regain $\left(\mathrm{g} \mathrm{g}^{-1}\right)$

${ }^{\mathrm{b}}$ Unreacted chlorine content $\left(\mathrm{mmol} \mathrm{g}^{-1}\right)$

c Total chlorine content (sum of ionic and covalently bonded) ( $\mathrm{mmol} \mathrm{g}^{-1}$ )

${ }^{\mathrm{d}}$ Ionic chlorine $\left(\mathrm{Cl}^{3}-\mathrm{Cl}^{2}\right)\left(\mathrm{mmol} \mathrm{g}{ }^{-1}\right)$

e Nitrogen content $\left(\mathrm{mmol} \mathrm{g}^{-1}\right)$

${ }^{\mathrm{f}}$ Hecker's ion exchange capacity $\left(\mathrm{mmol} \mathrm{g}^{-1}\right)$ 
The sorption processes were proceeded using a batch method. The resins were being shaken with a specific solution for $48 \mathrm{~h}$ at ambient temperature. Next, the polymers were separated by filtration. The concentration of the metals remaining in the solution was determined using Perkin-Elmer AAnalyst 200 atomic spectrophotometer. Then, sorption of noble metals, expressed in mg per gram of a dry resin, was calculated from the mass balance and presented in "Results and discussion".

\section{Column equilibrium}

The small $\left(5 \mathrm{~cm}^{3}\right)$ PE column has been applied for column equilibrium tests. Into the column, the most efficient resin, swollen in $0.001 \mathrm{M} \mathrm{HCl}$, received during present studies was introduced. The volume of the resin bead $\left(\mathrm{BV}_{\mathrm{s}}\right)$ was determined as $2.7 \mathrm{~cm}^{3}$.

Then, the multicomponent solution of $\mathrm{Au}(\mathrm{III}), \mathrm{Pt}(\mathrm{IV})$ and $\mathrm{Pd}(\mathrm{II})$ in $0.1 \mathrm{M} \mathrm{HCl}$ (0.24 mM of each metal) was passed through the column applying $10 \times 10^{-6} \mathrm{dm}^{3} \mathrm{~s}^{-1}$ flow rate until more than $5 \mathrm{dm}^{3}$ of the solution was flowed. The column effluent was collected in $15 \mathrm{~cm}^{3}$ portions using a fraction collector. Then, the concentrations of the $\mathrm{Au}(\mathrm{III}), \mathrm{Pt}(\mathrm{IV})$ and $\mathrm{Pd}(\mathrm{II})$ were determined and breakthrough curves were plotted.

\section{Results and discussion}

\section{Syntheses processes}

The Fig. 1 and the Table 2 display registered parameters of the modification processes, as well as determined characteristics of the resins $1 \mathrm{D}, 1 \mathrm{~S}$ and $1 \mathrm{~N}$; furthermore, Fig. 2 shows their FT-IR spectra recorded for an assessment of the received chemical structures.

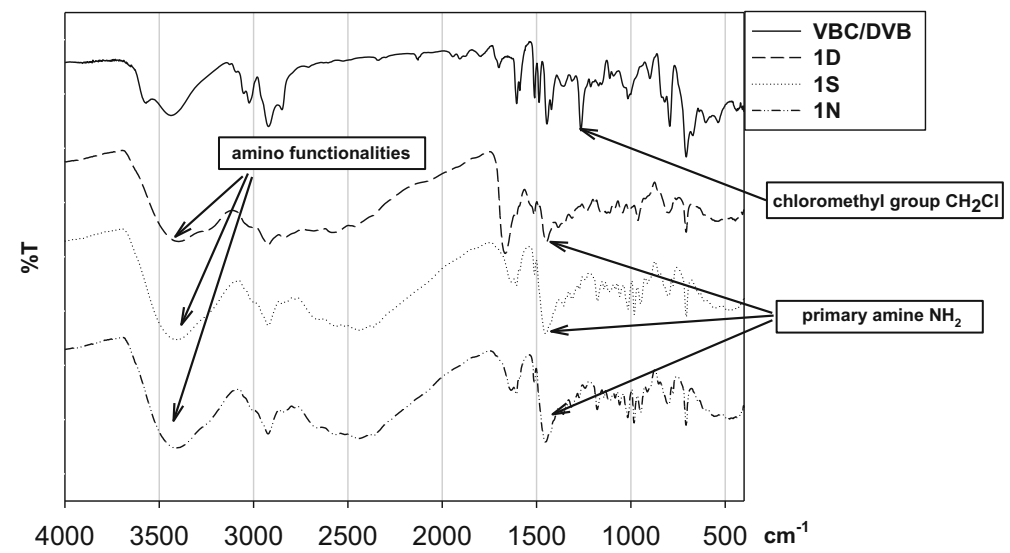

Fig. 2 FTIR spectra recorded for initial VBC/DVB copolymer and the resins 1D, 1S and 1N 
Based on the monitored reaction environment (Fig. 1) it could be seen that the synthesis of the resins $1 \mathrm{D}, 1 \mathrm{~S}$ and $1 \mathrm{~N}$ were proceeded in up to 75,90 and $85^{\circ} \mathrm{C}$, respectively. At the same time, the equipment allowed to register temperature of the vapors of the solvents, which proves that none of the systems has reached its boiling point. Moreover, the appliance of the low power allowed to provide a constant, uninterrupted functioning of the microwave field.

The differences between temperatures of the individual processes were minor. What is not surprising, they do result directly from dielectric properties of the specific solvent (Table 1). The reactions were not proceeded at a boiling point, and the environments were constantly affected by microwaves. Because of that, it could be stated that the properties of the resultant products will depend directly on interactions between microwave field and an organic solvent.

Each of the applied modification procedures did not allow for complete substitution of chlorine in initial VBC/DVB copolymer $\left(\mathrm{Cl}^{2}\right.$, Table 2). The effect is most probably attributed to the short reaction time $(15 \mathrm{~min})$, which means that the starting polymeric matrix suffers diffusional limitations in and out polymeric bead. The visible effect of the phenomenon was obviously intensified during rapid synthesis.

Ionic chlorine content $\left(\mathrm{Cl}^{4}\right.$, Table 2) reveals potential anion exchange "centres" on an anion exchange resin and arises directly from the determined nitrogen content (N, Table 2). That, in turn, defines the anion-exchange capacity estimated using Hecker's procedure $\left(Z_{\mathrm{H}}\right.$, Table 2$)$. While the results cannot be a base of the evaluation of the resins' sorption abilities, they provide an insight into modification procedure itself.

The greatest nitrogen content may be observed in the case of the resin $1 \mathrm{~N}$, synthesized in the presence of 1-methyl-2-pyrrolidone, NMP. Moreover, that resin reveals the greatest ionic chlorine content $\left(\mathrm{Cl}^{4}\right.$, Table 2$)$ as well as anion exchange capacity $\left(\mathrm{Z}_{\mathrm{H}}\right.$, Table 2$)$; therefore, it can be concluded that the microwave-assisted modification was the most efficient in system filled with NMP. The result was not expected, especially taking into account that the solvent does not possess a very good ability to absorb microwaves (Table 1). What is surprising, the resin $1 \mathrm{~N}$ reveals greater (than its equivalents $1 \mathrm{D}$ and $1 \mathrm{~S})$ unreacted chlorine amount $\left(\mathrm{Cl}^{2}\right.$, Table 2). Because of the fact, that syntheses were proceeded using the same VBC/ DVB copolymer, greater unreacted chlorine content should lead to lower amount of the introduced nitrogen, which did not happen. For that reason it is necessary to take a closer look at the recorded FT-IR spectra to verify the chemical structures of the resins.

\section{Evaluation of the FT-IR spectra}

In the Fig. 2 spectra of, the initial VBC/DVB copolymer, the resins $1 \mathrm{D}, 1 \mathrm{~S}$, and $1 \mathrm{~N}$, respectively, can be found. Sharp peak at $1263 \mathrm{~cm}^{-1}$ (VBC/DVB, Fig. 2) is responsible for $\mathrm{C}-\mathrm{H}$ interactions when simultaneously $\mathrm{Cl}$ is attached to the carbon. That proves the presence of the reactive chloromethyl groups. Then, as the result of modification that band almost disappeared and the new ones, responsible for aminomoieties, at $3426 \mathrm{~cm}^{-1}$ (resin 1D), $3426 \mathrm{~cm}^{-1}$ (resin 1S), and $3421 \mathrm{~cm}^{-1}$ (resin 1N) 
appeared. Based on that, the modification procedure was successful. As predicted, the resin 1D synthesized in DMF reveal sharp peak at $1660 \mathrm{~cm}^{-1}$ (1D, Fig. 2) responsible for the $-\mathrm{C}=\mathrm{N}-$ bonds, created due to the dimethyl formamide [8]. The effect, as described in our previous work [7] will affect final sorption capacity of the resin $1 \mathrm{D}$.

What is interesting, the peaks at $1447 \mathrm{~cm}^{-1}$ (resin 1D), $1447 \mathrm{~cm}^{-1}$ (resin 1S) and $1442 \mathrm{~cm}^{-1}$ (resin $1 \mathrm{~N}$ ), previously recognized as the indicator of the primary amino group $-\mathrm{NH}_{2}$ [7], differs with intensity from each other. The greatest band may be observed in the spectra recorded for the resin $1 \mathrm{~N}$ (Fig. 2), and the corresponding ones, registered for the resins $1 \mathrm{D}$ and $1 \mathrm{~N}$ are much weaker. The consequence of the phenomenon is displayed in the Fig. 3, where theoretical chemical structures of the resins are displayed.

The amine, DAH, applied for the synthesis procedures possess two primary amino groups, $-\mathrm{NH}_{2}$, on its "backbones". As the result of modification, one end of the DAH particle replaces chlorine placed in the chloromethyl groups, present in the initial VBC/DVB matrix. In effect, these new amino functionalities, on the one end, are methylene-bonded to the support, on the other, free primary amino groups may be observed (Fig. 3). However, due to the bis-amino functionality of the DAH, the synthesis process may reveal a different mechanism.

As concluded above, the modification of the VBC/DVB support in NMP was the most efficient. Despite lower conversion of the chloromethyl groups $\left(\mathrm{Cl}^{2}\right.$, Table 2)
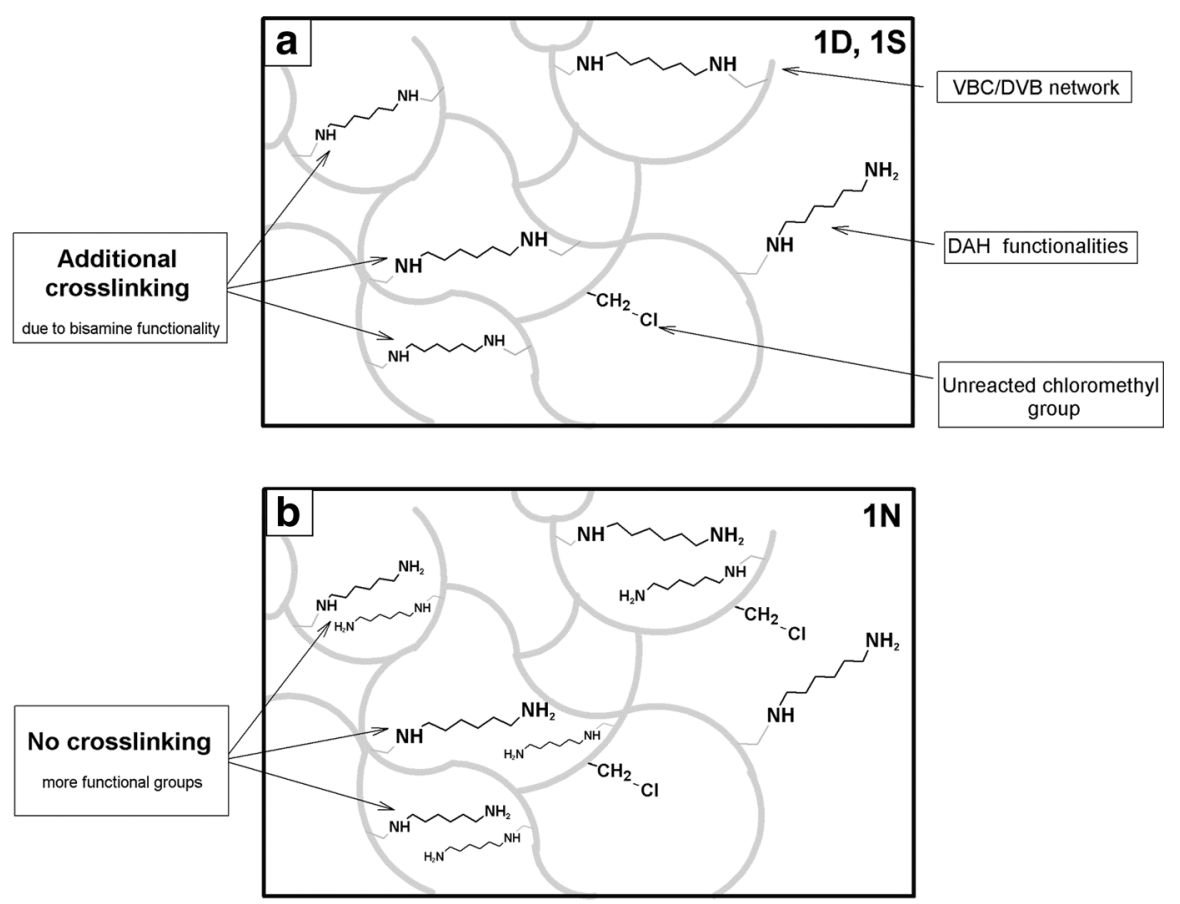

Fig. 3 The simplified proposal of the chemical structures of the resins (a) $1 \mathrm{D}, 1 \mathrm{~S}$ and (b) $1 \mathrm{~N}$ 
more nitrogen, responsible for the amount of functionalities, was introduced $(\mathrm{N}$, Table 2). Simultaneously, although more chlorine was displaced during synthesis of the resins $1 \mathrm{D}$ and $1 \mathrm{~S}\left(\mathrm{Cl}^{2}\right.$, Table 2), the process resulted with fewer functionalities (N, Table 2). The key to understand that phenomenon is hidden in the FT-IR spectra (Fig. 2). The data reveal presence of the great number of primary amines, but only in case of the resin $1 \mathrm{~N}$. In the structures of the resins $1 \mathrm{D}$ and $1 \mathrm{~N}$ much less $-\mathrm{NH}_{2}$ groups were registered. That means that more of the functionalities of the resins 1D and $1 \mathrm{~S}$ were participating in additional crosslinking reaction during modification process. As far as reduced amount of the primary amino groups should not significantly affect sorption behavior of the resins, the phenomenon entails a serious consequence. As proposed in Fig. 3a, additional crosslinking means, that one DAH particle will occupy more than one chloromethyl group, which strictly results with lower amount of the introduced functionalities. Of course, the resins $1 \mathrm{D}$ and $1 \mathrm{~N}$ reveal presence of the free primary amines (Fig. 2), which means that some of the functionalities were introduced as predicted.

To dispel doubts, the Kaiser's test was performed to ultimately prove the presence of the primary amino groups in the structure of the resins $1 \mathrm{D}, 1 \mathrm{~S}$ and $1 \mathrm{~N}$. $[9,15]$ The Kaiser's test is a simple colorimetric procedure that reveals these functionalities in investigated samples. As displayed in Fig. 4 the test gave a positive response for the sample of the $1 \mathrm{~S}$ polymer. Dark blue or even black color proves the presence of the $-\mathrm{NH}_{2}$ groups. The same result was recorded for the other resins, despite the fact, that some of the DAH functionalities were successfully introduced into structures of the resins $1 \mathrm{D}$ and $1 \mathrm{~S}$, the correlation between chlorine and nitrogen content (Table 2) as well as intensity of the peaks in the FT-IR spectra (Fig. 2) indicates that additional crosslinking occurred.

The phenomenon entails one more consequence. The resins $1 \mathrm{D}$ and $1 \mathrm{~S}$, that are post-crosslinked during modification process reveal much lower $0.001 \mathrm{M} \mathrm{HCl}$

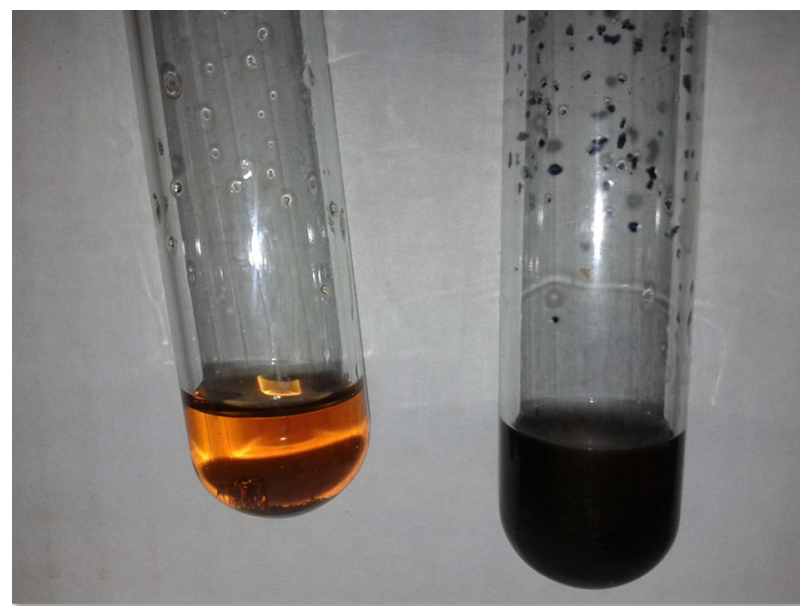

Fig. 4 The Kaiser's procedure performed on the resin 1S. The test tube on the left contains test solution, the one on the right is the solution with the sample added 
regain than the resin $1 \mathrm{~N}$. As a result, the sorption abilities of the polymer $1 \mathrm{~N}$ (synthesized using NMP) may be significantly boosted.

\section{Sorption of the noble metals}

Figure 5 gives the calculated sorption $\left(\mathrm{mg} \mathrm{g}^{-1}\right)$ of $\mathrm{Au}(\mathrm{III}), \mathrm{Pt}(\mathrm{IV})$ and $\mathrm{Pd}(\mathrm{II})$ from solution A (initial concentration $0.24 \mathrm{mM}$ of each metal).

Chlorocomplexes of gold, $\mathrm{AuCl}_{4}{ }^{-}$and platinum, $\mathrm{PtCl}_{6}{ }^{2-}$ were taken up with the best efficiency comparing to the sorption of palladium, $\mathrm{PdCl}_{4}{ }^{2-}$, that was removed with much lower quantity (Fig. 5). Moreover, the resins $1 \mathrm{~S}$ and $1 \mathrm{~N}$ revealed ability for complete removal of noble metals from diluted, hydrochloric systems. While sorption of $\mathrm{Au}(\mathrm{III}), \mathrm{Pt}(\mathrm{IV})$ and $\mathrm{Pd}(\mathrm{II})$ on the ion-exchange polymers $1 \mathrm{~S}$ and $1 \mathrm{~N}$ reached $100 \%$ (130 and $132 \mathrm{mg} \mathrm{g}^{-1}$, respectively), the removal of these metals on the $1 \mathrm{D}$ resin was only $55 \%\left(68 \mathrm{mg} \mathrm{g}^{-1}\right)$.

The effect has multiple sources, on the one hand, the resin reveals the lowest anion exchange capacity $\left(Z_{\mathrm{H}}\right.$, Table 2$)$, on the other, the lowest $0.001 \mathrm{M} \mathrm{HCl}$ regain. The lower value of its anion exchange capacity may be attributed to the creation of the $-\mathrm{C}=\mathrm{N}-$ bonds, that has been proven on the FT-IR spectra (Fig. 3). On the other hand, the low ability of the resin 1D to swell (determined by W, Table 2) means that the anion-exchange polymer 1D is able to uptake much less solution than its equivalents obtained using DMSO and NMP. The situation is completely different during synthesis of the same resin in the conventional conditions. Based on our previous work, [7] the resin 1D, if it had been obtained in course of a regular modification, should reveal twice greater $0.001 \mathrm{M} \mathrm{HCl}$ regain. That, without doubts should positively affect its ability to sorption. Nevertheless, because of the insufficient modification combined with worse ability to remove the noble metals it can be concluded that the DMF is inadequate for microwave-assisted synthesis of the ion-exchange resins.

In Fig. 6, the results of sorption from much more concentrated solution B (initial concentration $4.8 \mathrm{mM}$ ) can be found. The resin $1 \mathrm{~N}$ revealed the greatest maximum sorption capacity (634 $\mathrm{mg}$ of noble metals $\mathrm{g}^{-1}$, Fig. 6). However, the resin $1 \mathrm{~S}$ has sorbed the $\mathrm{Au}(\mathrm{III})$ preferably (the partition coefficient reached 9000). As predicted,

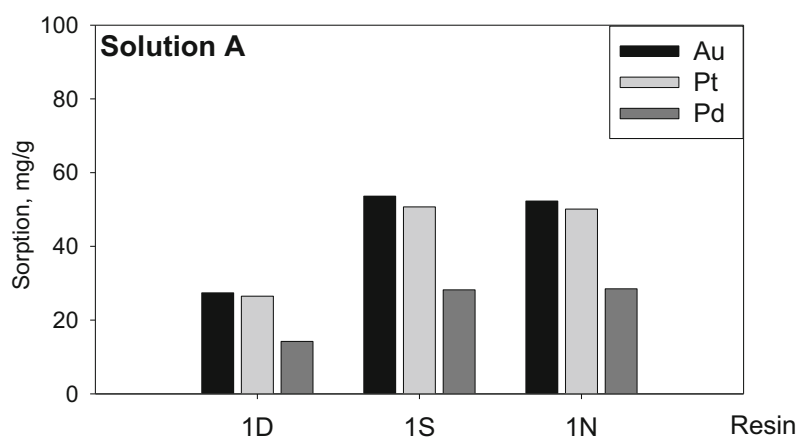

Fig. 5 Sorption of $\mathrm{Au}(\mathrm{III}), \mathrm{Pt}(\mathrm{IV})$ and $\mathrm{Pd}(\mathrm{II})$ from multicomponent $0.24 \mathrm{mM}$ solution in $0.1 \mathrm{M} \mathrm{HCl}$ 


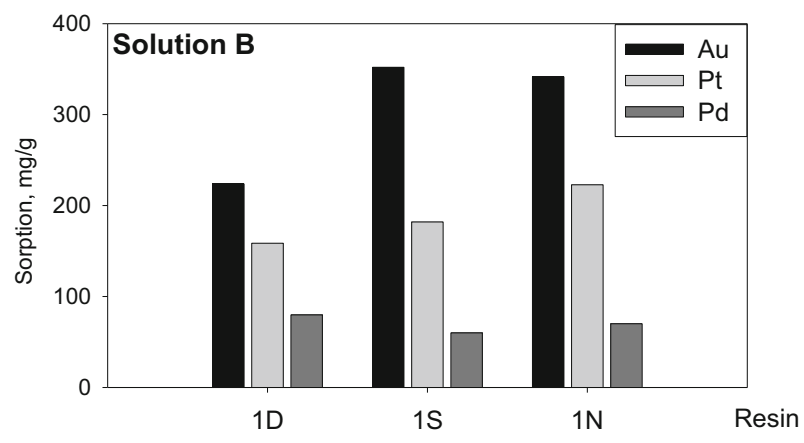

Fig. 6 Sorption of $\mathrm{Au}(\mathrm{III}), \mathrm{Pt}(\mathrm{IV})$ and $\mathrm{Pd}(\mathrm{II})$ from multicomponent $4.80 \mathrm{mM}$ solution in $0.1 \mathrm{M} \mathrm{HCl}$

the anion exchange resin $1 \mathrm{D}$, even in environment that provides complete saturation of its functionalities, reveals the lowest sorption capacity (462 mg of noble metals $\mathrm{g}^{-1}$, Fig. 6).

Based on the Figs. 5 and 6 it could be noticed that the sorption of Pd(II) is much lower than the uptake of $\mathrm{Au}(\mathrm{III})$ and $\mathrm{Pt}(\mathrm{IV})$. The effect is simply caused by molar mass of palladium (106 $\left.\mathrm{g} \mathrm{mol}^{-1}\right)$ that is significantly smaller compared to gold's $\left(197 \mathrm{~g} \mathrm{~mol}^{-1}\right)$ and platinum's $\left(195 \mathrm{~g} \mathrm{~mol}^{-1}\right)$. For that reason, sorption of $\mathrm{Pd}$, expressed in $\mathrm{mg} \mathrm{g}^{-1}$ will be always much smaller than the Au and Pt uptake, even if the amount (mmol) is comparable. After recalculating the values of sorption ( $\mathrm{mg} \mathrm{g}^{-1}$, Figs. 5, 6) it could be noticed, that sorption of Pt and Pd, expressed in mmol $\mathrm{g}^{-1}$ is always lower than the same value obtained for the removal of Au. The effect is caused just by the fact, that $\mathrm{PtCl}_{6}{ }^{2-}$ and $\mathrm{PdCl}_{4}{ }^{2-}$ are divalent and they require twice much anion exchange places than $\mathrm{AuCl}_{4}{ }^{-}$chlorocomplex.

Nevertheless, in any case, the removal of gold in form of $\mathrm{AuCl}_{4}{ }^{-}$was the most efficient, indicating, that the resins with functionalities derived from DAH synthesized in microwave field uptake $\mathrm{Au}(\mathrm{III})$ preferably.

The resin $1 \mathrm{~N}$ has the greatest ion-exchange capacity $\left(Z_{\mathrm{H}}\right.$, Table 2$)$ and its ability for sorption of noble metals is better than its equivalents $1 \mathrm{D}$ and $1 \mathrm{~S}$. It can only mean, that the great $0.001 \mathrm{M} \mathrm{HCl}$ regain $\left(1.72 \mathrm{~g} \mathrm{~g}^{-1}\right.$, Table 2) as well as great amount of the introduced functionalities makes the polymer $1 \mathrm{~N}$ just more efficient.

The total sorption capacity of the resins $1 \mathrm{D}, 1 \mathrm{~S}$ and $1 \mathrm{~N}$ expressed in mol g $\mathrm{g}^{-1}$ is $2.70,3.28$ and $3.54 \mathrm{mmol}$ of noble metals $\mathrm{g}^{-1}$, respectively. These values are quite similar to the determined Hecker's anion exchange capacity and any differences may be correlated with the influence of the $0.001 \mathrm{M} \mathrm{HCl}$ regain.

The anion exchange resins synthesized in the present studies are based on the same VBC/DVB copolymer as these presented in our previous works cited in the references $[7,9,16]$. For that reason, it is possible to access the efficiency of the polymers obtained using different functionalities and procedures.

It has been previously recognized that the resins modified with 1-methyl piperazine reveal total capacity of $\mathrm{Au}(\mathrm{III}), \mathrm{Pt}(\mathrm{IV})$ and $\mathrm{Pd}(\mathrm{II})$ sorption at $796 \mathrm{mg} \mathrm{g}^{-1}$ [9]. At the same time, the most efficient resin $1 \mathrm{~N}$ described in the present work uptakes $634 \mathrm{mg} \mathrm{g}^{-1}$ of the metals. The result is lower, however, considering the 
time required for completing the synthesis procedure (15 min. vs $24 \mathrm{~h} \mathrm{[9])} \mathrm{the}$ sorption capacity is more than satisfactory. Moreover, the resin $1 \mathrm{~N}$ is more efficient than its equivalent modified in regular conditions using 4-aminopyridine [16] which maximum sorption capacity has been determined as $537 \mathrm{mg} \mathrm{g}^{-1}$.

The resins presented in present studies as well as these synthesized in microwave field described in our previous work [7] are far from being as much efficient as the resins based on chloromethylated styrene-divinylbenzene copolymers (their sorption of gold capacity reaches $1060 \mathrm{mg} \mathrm{g}^{-1}$ [17]). However, their other advantages should not be omitted. On the one hand, application of VBC/DVB copolymer allows to exclude the usage of carcinogenic alkylating reagents required for preparation of S/DVB matrixes [18]. On the other, application of microwave-assisted process allows for serious reduction of the time required for the synthesis, being simultaneously much less resource intensive, and, still, the efficiency of the obtained anion exchange resins is more than satisfactory.

\section{Dynamic equilibrium tests}

The Fig. 7 displays registered breakthrough curves obtained during dynamic sorption tests. The graph expresses as unified ratio of initial/equilibrium concentration of the $\mathrm{Au}, \mathrm{Pt}$ and $\mathrm{Pd}$ in function of a volume of the flowed solution set together with volume of the resin's bead $\left(2.7 \mathrm{~cm}^{3}, \mathrm{BV}_{\mathrm{s}}\right)$.

The maximum dynamic sorption capacities of the gold were determined as $344 \mathrm{mg} \mathrm{Au} \mathrm{g}^{-1}$, platinum $226 \mathrm{mg} \mathrm{Pt} \mathrm{g}^{-1}$ and palladium $74 \mathrm{mg} \mathrm{Pd} \mathrm{g}^{-1}$. The results comply with these obtained using batch procedure. However, breakthrough curves (Fig. 7) are revealing some interesting facts. Over $5 \mathrm{dm}^{3}$ of $0.25 \mathrm{mM}$ metals' solution was needed to completely saturate the resin $1 \mathrm{~N}$. The first metal that has broke the column was $\mathrm{Pd}(\mathrm{II})$ despite the fact, that $\mathrm{Pt}(\mathrm{IV})$ and $\mathrm{Au}(\mathrm{III})$ were still being

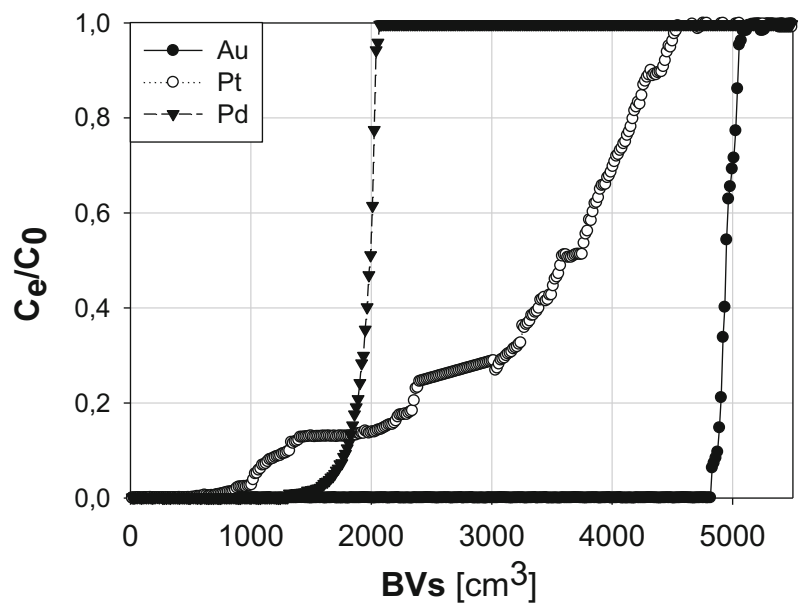

Fig. 7 Breakthrough curves registered during column studies on sorption of $\mathrm{Au}, \mathrm{Pt}$ and $\mathrm{Pd}$ from multicomponent $0.24 \mathrm{mM}$ solution in $0.1 \mathrm{M} \mathrm{HCl}$ on the resin $1 \mathrm{~N}$ 
sorbed i.e., there were still anion exchange centres available (Fig. 7). The fact means, that the resin $1 \mathrm{~N}$ reveals greater affinity towards platinum and gold, extracting them more efficiently. Based on Fig. 7 it could be stated that gold is the most competitive towards rest of the metals and as a result, it appeared as the last one in the effluent reaching the greatest sorption capacity.

No clear breakthrough of platinum is observed (Fig. 7). Instead, concentration of $\mathrm{Pt}(\mathrm{IV})$ was gradually increasing in the outflow during the process until it reached equilibrium. Shape of its breakthrough curve indicate that separation of platinum from gold and palladium could be difficult. The similar effect has been observed during our previous studies [9] and it can be explained by octahedral shape of the $\mathrm{PtCl}_{6}{ }^{2-}$ chlorocomplex. It is often observed that flat and rectangular $\mathrm{Au}(\mathrm{III})$ and $\mathrm{Pd}(\mathrm{II})$ species are most easily extracted during sorption processes, while more complicated $\mathrm{PtCl}_{6}{ }^{2-}$ usually makes some difficulties [9].

\section{How organic solvents influence the usability of the resins?}

It was expected that the ability to absorb the microwaves (Table 1) will be a major factor that will define the efficiency of a resin obtained in a specific solvent. Based on that, appliance of the solvent with outstanding ability to transform the microwave radiation into heating energy (DMSO, Table 1), should result with a product with distinctive properties. That did not happen.

The selection of the best and the worst organic solvent for microwave-assisted process, in present studies, may be obvious. To justify the choice, all of the relevant parameters revealed in the present studies are displayed in the Table 3.

The N,N-dimethyl formamide, DMF, should not be used for microwave-assisted synthesis of anion-exchange resins. It promotes transformation of the chloromethyl groups, present in the VBC/DVB polymeric matrix into the $-\mathrm{C}=\mathrm{N}-$ bonds. As a result, these functionalities did not react with $\mathrm{DAH}$, significantly decreasing the sorption capacity of the resin 1D.

Based on the $0.001 \mathrm{M} \mathrm{HCl}$ regain (W, Table 2) the solvents DMF and DMSO caused, under influence of the microwave field, additional crosslinking of the polymeric matrix. In effect, less anion-exchange functionalities were introduced which reflected on the lower ion-exchange as well as sorption capacity.

Despite the fact, that NMP reveals not perfect dielectric properties and its ability to transform the microwaves into heat is middling (Table 1), the resin $1 \mathrm{~N}$ showed the greatest sorption capacity. The solvent (NMP) caused, that the chloromethyl

Table 3 Summary of the influence of the organic solvents

\begin{tabular}{llll}
\hline Issue & DMF (1D) & DMSO (1S) & NMP (1N) \\
\hline Additional crosslinking during modification & + & + & - \\
Creation of the $-\mathrm{C}=\mathrm{N}-$ bonds during modification & + & - & - \\
Efficiency of the modification & Bad & Good & Very good \\
Regain of the $0.001 \mathrm{M} \mathrm{HCl}$ & Bad & Middling & Very good \\
Sorption capacity & Bad & Good & Very good \\
\hline
\end{tabular}


groups was efficiently used; furthermore, in that environment more functionalities might have been introduced (Table 2).

Because the synthesis of the resin $1 \mathrm{~N}$ proceeded without undesired transformations, resulting with great anion exchange capacity of the anion-exchanger, it can be concluded, that the solvent NMP should be preferred in planning microwaveassisted synthesis of functional resins.

\section{Conclusion}

In the present work the series of anion exchange resins was synthesized. The processes were differed using selected organic solvents. The resins $1 \mathrm{D}, 1 \mathrm{~S}$ and $1 \mathrm{~N}$ were obtained in the microwave field using DMF, DMSO and NMP as the reaction environment, respectively. Then, the polymers were employed in processes of sorption of $\mathrm{Au}(\mathrm{III}), \mathrm{Pt}(\mathrm{IV})$ and $\mathrm{Pd}(\mathrm{II})$ from hydrochloric systems.

Based on the observations it is possible to access the usability of the selected solvents:

- The NMP turned out to be the best organic solvent for microwave-assisted synthesis of anion-exchange resins. The reaction in that environment did not reveal additional processes that negatively affect sorption capacity. The resin $1 \mathrm{~N}$ showed the greatest amount of the introduced functionalities, the most efficient conversion of the chloromethyl groups in the initial polymeric matrix as well as the greatest ion-exchange capacity. As a result, the anion-exchange polymer revealed sorption of noble metals $634 \mathrm{mg} \mathrm{g}^{-1}$.

- The DMSO, due to its dielectric properties allows to proceed modification processes efficiently. The conversion of the chloromethyl groups as well as the amount of the introduced functionalities was satisfactory. Nevertheless, despite the "microwave-compatibility" of the DMSO, the resin $1 \mathrm{~S}$ revealed additional crosslinking of the polymeric matrix, which decreased the anion exchange capacity as well as $0.001 \mathrm{M} \mathrm{HCl}$ regain. As a result, the sorption of the noble metals on the anion-exchange resin $1 \mathrm{~S}$ was $594 \mathrm{mg} \mathrm{g}^{-1}$.

- The DMF is not suitable for microwave-assisted processes. The solvent promotes creation of $-\mathrm{C}=\mathrm{N}-$ bonds instead of amino functionalities; furthermore, extent of crosslinking due to bisamine functionality was observed. As a result, the anion-exchange resin revealed the smallest sorption capacity $462 \mathrm{mg}$ of noble metals $\mathrm{g}^{-1}$.

Furthermore it can be concluded that:

- The resins $1 \mathrm{~S}$ and $1 \mathrm{~N}$ are suitable for complete removal of noble metals from solutions in $0.1 \mathrm{M} \mathrm{HCl}$.

- All of the resins are taking up gold preferably.

- The properties of the anion-exchange resins are not strictly dependent on the dielectric properties of the reaction environment. 
Acknowledgments The work has been financed by a statutory activity subsidy from the Polish Ministry of Science and Higher Education for the Faculty of Chemistry of Wroclaw University of Technology.

Open Access This article is distributed under the terms of the Creative Commons Attribution 4.0 International License (http://creativecommons.org/licenses/by/4.0/), which permits unrestricted use, distribution, and reproduction in any medium, provided you give appropriate credit to the original author(s) and the source, provide a link to the Creative Commons license, and indicate if changes were made.

\section{References}

1. Loupy A (2002) Microwaves in organic synthesis. Wiley, Weinheim

2. Clark DE, Folz DC, Folgar CE, Mahmoud MM (2005) Microwave solutions for ceramic engineers. American Ceramic Society, Westerville

3. Committee on microwave processing of materials: an emerging industrial technology (1994) Microwave processing of materials. National Academy Press, Washington D.C.

4. Elkady MF, Mahmoud MM, Abd-El-Rahman HM (2011) Kinetic approach for cadmium sorption using microwave synthesized nano-hydroxyapatite. J Non-Cryst Solids 357:1118

5. Mondal BC, Das AK (2001) Microwave-assisted synthesis of a new chelating resin containing 2-aminothiophenyl S-acetic acid and its application to the determination of lead. React Funct Polym $53: 45$

6. Bogdał D (2005) Microwave-assisted organic synthesis. One hundred reaction procedures. Elsevier, Oxford

7. Jermakowicz-Bartkowiak D, Cyganowski P (2015) Effect of microwave radiation on the synthesis of ion exchange resins: a comparative study. Solvent Extr Ion Exch 33:510

8. Alexandratos SD, Zhu X (2003) Amination of poly(vinylbenzyl chloride) with $N, N$-dimethylformamide. Macromolecules 36:3436

9. Cyganowski P, Jermakowicz-Bartkowiak D (2014) Piperazine functionalized resins for $\mathrm{Au}(\mathrm{III})$, Pt(IV), and Pd(II) sorption. Sep Sci Technol 49:1689

10. Wolska J, Bryjak M (2011) Preparation of polymeric microspheres for removal of boron by means of sorption-membrane filtration hybrid. Desalination 283:193

11. Kjeldahl JZ (1883) A new method for the determination of nitrogen in organic bodies. Anal Chem $22: 366$

12. Jermakowicz-Bartkowiak D, Kolarz BN (2011) Poly(4-vinylpyridine) resins towards perrhenate sorption and desorption. React Funct Polym 71:95

13. Vogel AI (1978) Handbook of quantitative inorganic analysis. Longman, London

14. Hecker H (1974) Beitrag zur basizitätsprüfung von stark basischen anionenaustauschern. J Chromatogr 102:135

15. Kaiser E, Colescott RL, Bossinger CD, Cook P (1970) Color test for detection of free terminal amino groups in the solid-phase synthesis of peptides. Anal Biochem 34:595

16. Cyganowski P, Jermakowicz-Bartkowiak D (2013) Recovery of precious metals on ion-exchange polymers. Chemik 37:321

17. Yi-Yong C, Xing-Zhong Y (1994) Synthesis and properties of 1-(2-aminoethyl)piperazine resin used in the sorption of the platinum group and gold ions. React Pol 23:165

18. Concha-Barrientos M, Nelson DI, Driscoll T et al (2004) Selected occupational risk factors. In: Ezzati M (ed) Comparative quantification of health risks. World Health Organization, Geneva, pp 1651-1802 